\title{
A Double-Blind, Randomized, Placebo-Controlled Nutritional Study Using an Insoluble Yeast Beta-Glucan to Improve the Immune Defense System
}

\author{
Hans-Joachim Graubaum, Regina Busch, Heike Stier, Joerg Gruenwald
}

Analyze \& Realize AG, Berlin, Germany.

Email: hstier@analyze-realize.com

Received February $21^{\text {st }}, 2012$; revised April $5^{\text {th }}, 2012$; accepted April $13^{\text {th }}, 2012$

\begin{abstract}
Purpose: In a placebo-controlled, double-blind, randomized clinical trial, the effect of an insoluble yeast beta-glucan preparation on the incidences of common colds and its effect on common cold symptoms were compared to placebo. Methods: 100 healthy participants with recurring infections were randomly assigned to receive either placebo or yeast beta-glucan (Yestimun ${ }^{\mathbb{B}} ; \mathrm{n}=50$ each group) over a period of 26 weeks. The subjects had to document each common cold episode in a diary, and rate 6 predefined infections symptoms on a 3-point rating scale during an infection period, resulting in an infection score. The common cold episodes were confirmed by the investigators. Results: A total of 171 common cold episodes were documented. Of these, 76 were experienced by 38 subjects in the beta-glucan group and 96 were experienced by 48 subjects in the placebo group $(p=0.406)$. The beta-glucan group had significantly more subjects without incidences of common cold than the placebo group $(15.6 \%$ vs $2.0 \% ; p=0.019)$. During the most intense infection season (first 13 weeks of the study), the beta-glucan group had significantly less infections compared to placebo $(p=0.02)$. Beta-glucan significantly reduced the typical cold symptoms ("sore throat and/or difficulty swallowing", "hoarseness and/or cough" and "runny nose") as opposed to placebo. Conclusion: The present study demonstrates a prophylactic effect of yeast beta-glucan on the occurrence of common colds as opposed to placebo. In addition, when these episodes occurred, they were from the beginning less pronounced and subsided faster.
\end{abstract}

Keywords: Randomized Placebo-Controlled Study; Insoluble Yeast Beta-Glucan; Common Cold; Immune System

\section{Introduction}

Beta-glucans are natural polysaccharides containing Dglucose subunits, linked by beta-glycosidic bindings. They are found in cell walls of bacteria, yeast, fungi, algae, but also in the walls of grain and other plants.

Initial studies investigating the inmunomodulatory activity of beta-glucan were performed in 1956. In that study, a chitin/beta-glucan preparation led to an unspecific stimulation of the immune system, leading to an increased anti-bacterial and anti-viral activity after application of the substance to rabbits and guinea pigs [1]. Since these early studies, numerous publications and patents on the effects and mode of actions of beta-glucans have been published.

Beta-glucan is neither synthesized by the human organism nor is it a component of the human body, therefore it is identified by the immune system as foreign, leading to activation of both the innate as well as the adaptive immune response [2]. Even though the mode of action is not fully understood, several effects of betaglucan on various parts of the immune system have been demonstrated. It has been found that Dectin-1 and tolllike receptors (TLR), expressed on macrophages, neutrophils and dendritic cells are responsible at least partially for the innate immune response, leading to activated immune cells and the release of various cytokines. Via binding to the activated complement receptor 3 (CR3), cell lysis or monoclonal antibody reaction might be potentiated by beta-glucans $[2,3]$.

Many of these effects found in in vitro investigations have been confirmed in vivo. Studies on breeding cattle demonstrated a positive effect of beta-glucans on their ability to activate their immune system. Fleischer et al. (2001) investigated the effect of $(1,3)-(1,6)-\beta$-D-glucan on the immune system of chicks and breeding sows [4]. During this study, the regular animal feed was mixed with yeast beta-glucan. Several immune parameters (such as number of monocytes, activation status of macrophages and monocytes by the indicator neopterin, im- 
munoglobulin A etc.) were measured to determine the immune status under various conditions (under physical exertion, growth phases, pregnancy, birth and lactation). As a result, they found that yeast beta-glucan, when mixed with the regular feed, led to increased resistance against Escherichia coli in the growing chick, the breeding sows as well as in their offspring.

In another study, $(1,3)-(1,6)-\beta$-D-glucan was given to piglets and the immunological effects were investigated in vivo as well as in vitro. In vivo $(1,3)-(1,6)-\beta$-D-glucan led to a reduced production of IL- 6 and $\operatorname{TNF} \alpha$, and a simultaneous increase of IL-10. The reduction of IL-6 und TNF $\alpha$ had also been observed in vitro [5].

All these studies demonstrate that beta-glucan strengthens the immune function in general. It has been shown that beta-glucan is able to improve the resistance against various invading pathogens. Animal studies showed positive effects on the defense against infection by Staphylococcus aureus, Escherichia coli, Candida albicans, Pneumocystis carinii, Listeria monocytogenes, Leishmania donovani, and influenza virus but also against anthrax challenge (for review see [6]).

Beta-glucans are not a unique class, but occur in different molecular weight and structures. Depending on the origin and structure of the molecule, they differ in their biological specificity as well as in their efficacy. For the biological activity, the molecular weight as well as the branching structure seems to be important [6]. It seems that the more complex the structure, the more potent are the immunmodulatory effects [7]. Highly polymer betaglucans with a molecular weight of $100,000 \mathrm{Da}-200,000$ $\mathrm{Da}$ expressed the highest immune stimulating activity, whereas fractions of very short beta-glucans between $5000 \mathrm{Da}-10,000 \mathrm{Da}$, extracted from the same organism, exhibited no activity [8]. Further investigations showed that particles with $\beta-(1,6)$ branching together with $\beta-(1,3)$ branching are more effective than $\beta-(1,3)$ branching alone [9]. It seems that at least for common cold prevention, soluble $(1,3)-(1,4)-\beta$-glucan prepared from oat is not effective [10]. Therefore it is very important to clearly differentiate between results obtained from various molecules.

In this study, the insoluble $(1,3)-(1,6)-\beta$-glucan made from brewers' yeast (Saccharomyces cerevisiae: Yestimun $^{\circledR}$, previously called Biolex Beta-HP), was used. The positive effects of this specific preparation on the immune system have been confirmed in various in vitro studies [11-13]. Herein the common cold was used as a model system to show that the insoluble beta-glucan preparation activates the human defense system against various pathogens. The common cold is a well-known and, in most cases, harmless infection, typically affecting adults 2 - 5 times per year [14]. The aim of this study was to show that intake of a functional food composed of beta-glucan led to less infections of reduced intensity in the general population. The results of this human trial with this product are presented here.

\section{Methods}

\subsection{Study Design}

This nutrition study was conducted as a prospective, multi-centric, randomized, double-blind, placebo-controlled study in healthy outpatient subjects, with recurring common colds, in Berlin (Germany) between November 2006 and September 2007. It was conducted in the practices of five physicians. The effect of an insoluble yeast beta-glucan preparation on the incidences of common colds was compared to placebo. The study was performed according to the declaration of Helsinki/Hong Kong 1989/Somerset 1996 as well as the ICH-GCP guidelines (CPMP/ICH/135/95).

\subsection{Study Population}

One hundred healthy subjects with recurring common colds were included in this nutritional study. They had to meet the inclusion criteria: age $\geq 18$, written consent to participate, and at least three infections within the last six months. The exclusion criteria were as follows: allergic rhinitis or asthma, known sensibility to one of the ingredients of the study product, fever $\left(\geq 39^{\circ} \mathrm{C}\right)$, severe organ or systemic disorders, stomach or intestinal diseases, intake of preparations that can influence the study outcome, such as immunosuppressives or common cold therapeutics, bacterial tonsillitis, incidence of alcohol, medication or drug abuse, cancer or being HIV positive, pregnancy or nursing, participation in a clinical study within the previous 30 days, or problems with compliance or following the protocol due to language difficulties.

All of the screened subjects met the inclusion criteria and did not violate the exclusion criteria and were randomized into this nutritional trial. They represent the intention to treat (ITT) population (see Figure 1). In total, six subjects withdrew from the study. One case was due to insufficient efficacy (placebo group $=\mathrm{PG}$ ), two were without giving a reason (active group $=A G$ ), one was due to a move (AG), one stated aversion to capsules (AG), and one (AG) was due to loss of motivation. Therefore, in total 94 subjects completed the entire study. Of these 94 subjects who completed the entire study, nine were excluded from the per protocol (PP) analysis due to poor compliance (4 PG, $5 \mathrm{AG}$ ) resulting in $\mathrm{n}=85$ subjects (41 AG, $44 \mathrm{PG}$ ).

Of the 100 subjects taken into the study, 58 were 


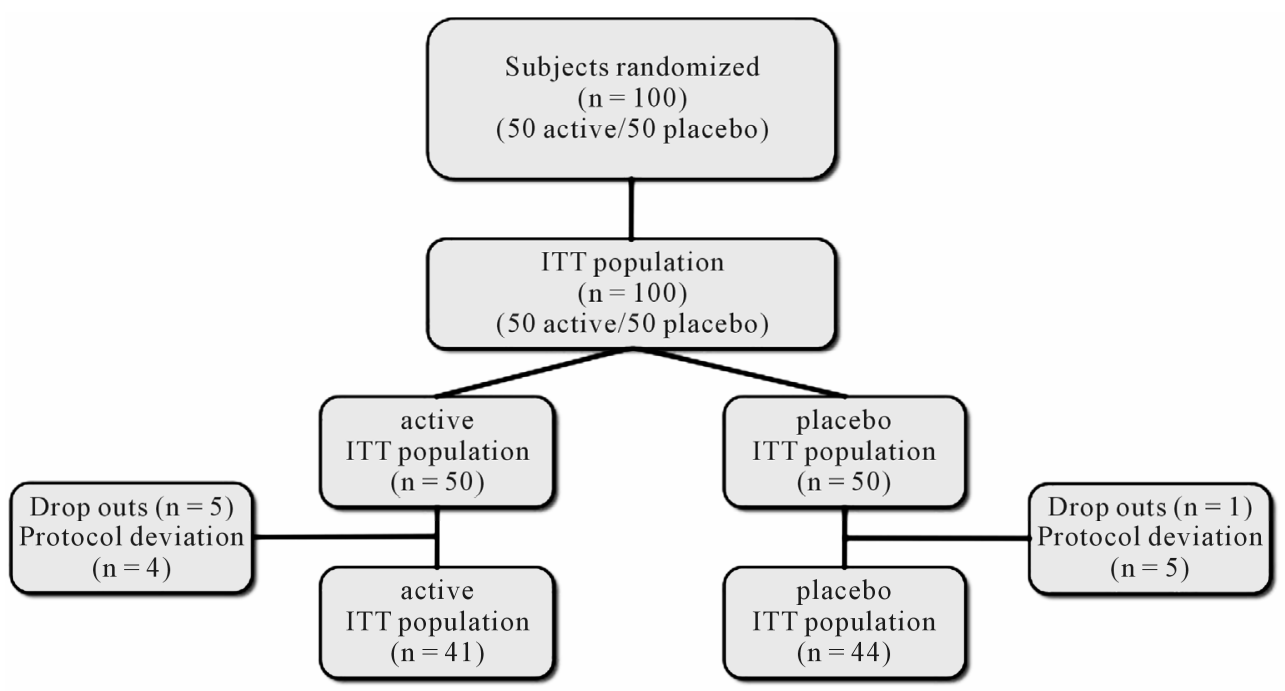

Figure 1. Schematic representation of the study population. ITT: intention to treat; PP: per protocol.

women and 42 were men. All subjects reported that they had experienced at least three cold episodes in the six months prior to beginning the study. The mean height, weight and body mass index (BMI) for the subjects are provided in Table 1. All the study participants were classified as Caucasian. With respect to these baseline characteristics, there were no statistically significant differences between the beta-glucan group and placebo group (Table 1).

\subsection{Interventions}

The study participants were instructed to take two times daily one capsule of the active treatment (one capsule contains $450 \mathrm{mg}$ insoluble yeast beta-glucan; Yestimun ${ }^{\circledR}$, formerly called Biolex Beta-HP; Leiber, Germany) or placebo (microcrystalline cellulose) over a period of 26 weeks (prophylactic treatment). The beta-glucan preparation is an insoluble $(1,3)-(1,6)-\beta$-glucan made from brewers' yeast (Saccharomyces cerevisiae), with a purity of min. $85 \%$ on dry matter. (branching factor approximately: 1,3 (backbone):1,6 (side chain):1,3/1,6 (branching $=10: 1: 0.6)$. During the study period, three regular visits (V1 at baseline, V2 after 12 - 14 weeks, and V3 after 25 - 27 weeks) were performed in the practices of one of the five investigators. In case of a beginning common cold episode, the participant took for a period of five days two times daily two capsules of the assigned product (active treatment or placebo) and had to visit the investigator for the first episode visit. After these five days (day six), the participant had to consult once more the investigator for the second episode visit. For each occurring infection, the same procedure was performed. Thus, the total number of visits per participant varies depending on the number of infection episodes.
Table 1. Characteristics of the participants at baseline.

\begin{tabular}{cccc}
\hline & \multicolumn{3}{c}{ Study population } \\
\cline { 2 - 4 } & Beta-glucan $(n=50)$ & Placebo $(n=50)$ & $p$-value \\
\hline Age (years) & $45.6 \pm 16.9$ & $47.1 \pm 16.4$ & 0.629 \\
Sex $(\mathrm{m} / \mathrm{f})$ & $22 / 28$ & $20 / 30$ & 0.685 \\
Height $(\mathrm{cm})$ & $173.5 \pm 9.1$ & $171.5 \pm 9.1$ & 0.394 \\
Body weight $(\mathrm{kg})$ & $74.4 \pm 13.8$ & $74.6 \pm 17.1$ & 0.929 \\
BMI kg/m & $24.7 \pm 3.8$ & $25.3 \pm 5.2$ & 0.672 \\
\hline
\end{tabular}

Participants with a common cold episode at the beginning of the nutritional study (for no longer than $24 \mathrm{~h}$ ), started with the two capsules two times daily for five days treatment, followed by the prophylactic phase (regular intake two times daily one capsule).

All the subjects were encouraged to continue their normal background diet during the duration of the study.

\subsection{Outcome Measures}

\subsubsection{Primary Outcome}

The primary objective of this double-blind, randomized, placebo controlled nutritional study was to prove a significant reduction of common cold episodes by the insoluble yeast beta-glucan preparation, as compared to placebo, in subjects with an increased risk of common cold infections, over a period of 26 weeks. All the common cold episodes had to be documented in a diary and confirmed by an investigator.

\subsubsection{Secondary Outcome}

The secondary objective was to prove a significant reduction of common cold symptoms by the yeast insolu- 
ble beta-glucan as compared to placebo within the first five days of each common cold episode. During the infection episode, the participants had to rate six predefined common cold symptoms (general malaise, headache and/or pain in the limbs, "sore throat and/or difficulty swallowing", "hoarseness and/or cough", "runny nose", insomnia due to common cold) on a rating scale (0 points $=$ complaint free, 1 point $=$ weak symptoms, 2 points $=$ strong symptoms) and had to document them in his/her dairy. By summation of the scores of the individual symptoms, a sum of scores (=total score) was calculated.

\subsubsection{Concurrent Variable}

As a concurrent variable, the efficacy of the investigational product was evaluated by the subjects and the investigator at the end of each common cold episode as "very good" "good", "moderate", or "poor".

\subsubsection{Safety Assessment}

The safety and tolerability of the product was evaluated by the documentation of adverse events as well as by the global evaluation of the tolerability by the investigator and the subject at the end of the study.

\subsection{Randomization/Blinding}

The human study was conducted double-blind; neither the participants nor the investigators knew which study participants had the active or placebo treatment.

Group assignment to the two study groups was performed during the first examination (baseline) randomly and double-blind, if the inclusion and exclusion criteria were met. The randomization was performed by block randomization (block size 4) using the randomization scheme EDGAR (Experimental Design Generator and Randomizer). The issued containers comprising the active treatment or the placebo product were pre-numbered (externally) according to the randomization. The random code remained blinded until the final closing of the database.

\subsection{Statistical Methods/Sample Size Calculation}

All the variables contained in the data collection were presented descriptively using their statistical key data or their frequency distribution and statistically analyzed in view of the group specific differences ( $\mathrm{p}_{\gamma^{2}}$-value). Ordinal and metric data were evaluated using the U-test as well as the Mann-Whitney U-test for independent sample testing ( $\mathrm{p}_{\mathrm{u}}$-values), and the Wilcoxon-test for dependent sample testing. The t-test was additionally used for metric data ( $\mathrm{p}_{\mathrm{t}}$-values), whereby testing for equality of variances in comparison to independent or paired data was performed by means of an F-test. All statistical analyses were carried out on an intention-to-treat (ITT) and per protocol (PP) basis with SPSS. Values of $p<0.05$ were considered significant. Means are given with standard deviation (SD).

A sample size calculation was not performed, due to missing preliminary data. The number of subjects was adjusted to 50 subjects each group.

\section{Results}

\subsection{Total Number of Infections}

For the primary objective (total number of infections), the ITT population consisted of only 94 subjects, due to six drop-outs, which had a shorter study period and therefore unavoidably less total infections (see Figure 1).

Within the ITT population, a total of 171 cold episodes occurred in the prophylactic period of 26 weeks (Table 2). Of these, 76 affected 38 subjects of the active group (AG; 44.4\%) and 95 affected 48 subjects in the placebo group (PG; 55.6\%). Even though the AG showed 25\% less infection episodes than the $\mathrm{PG}$, and a total of seven subjects $(15,6 \%)$ of the AG compared to only one of the PG (2\%) had no common cold episode during the entire study period $\left(\mathrm{p}_{\gamma^{2}}=0.019\right)$, the difference in the frequency distribution showed only a tendency towards significance between the two treatment groups (ITT: $\mathrm{p}_{\chi^{2}}$ $=0.094 ;$ PP: $p_{2^{2}}=0.060$ ), and no significant difference in the total number of episodes (ITT: $\mathrm{p}_{\mathrm{u}}=0.406$; PP: $\mathrm{p}_{\mathrm{u}}=$ 0.614 , respectively).

However, if the same data analysis was made for the first half (13 weeks) of the prophylactic period, from

Table 2. Number of common cold episodes during the entire study (26 weeks) in the intention to treat (ITT) and per protocol (PP) population.

\begin{tabular}{ccccc}
\hline \multirow{2}{*}{$\begin{array}{c}\text { Number of } \\
\text { episodes }\end{array}$} & \multicolumn{1}{c}{ AG $($ ITT $\mathrm{n}=45 / \mathrm{PP} \mathrm{n}=41)$} & $\mathrm{PG}(\mathrm{ITT} \mathrm{n}=49 / \mathrm{PP} \mathrm{n}=44)$ \\
\cline { 2 - 5 } & Number & $\%$ & Number & $\%$ \\
\hline 0 & $7 / 7$ & $15.6 / 17.1$ & $1 / 1$ & $2.0 / 2.3$ \\
1 & $11 / 10$ & $24.4 / 24.4$ & $18 / 18$ & $36.7 / 40.9$ \\
2 & $19 / 17$ & $42.2 / 41.5$ & $18 / 16$ & $36.7 / 36.4$ \\
3 & $5 / 4$ & $11.1 / 9.8$ & $10 / 8$ & $20.4 / 18.2$ \\
4 & $3 / 3$ & $6.7 / 7.3$ & $1 / 0$ & $2.0 / 0$ \\
7 & $0 / 0$ & $0 / 0$ & $1 / 1$ & $2.0 / 2.3$ \\
Total & $76 / 68$ & $100 / 100.1$ & $95 / 81$ & $99.8 / 100.1$ \\
$\mathrm{p}_{u}$ & \multicolumn{5}{c}{$0.406 / 0.614$} \\
$\mathrm{p}_{\chi^{2}}$ & \multicolumn{5}{c}{$0.094 / 0.060$} \\
\hline
\end{tabular}

$\mathrm{p}_{\mathrm{u}}$ : $\mathrm{p}$-value for total number of episodes; $\mathrm{p}_{z^{2}}: \mathrm{p}$-value for distribution; AG: active group; PG: placebo group. 
November to March, when most of the common cold episodes occurred, the AG had significantly less infections as compared to PG $\left(p_{u}=0.020\right.$; see Tables 3 and 4).

\subsection{Reduction of the Common Cold Symptoms (Secondary Objective)}

To evaluate the effect of beta-glucan on the common cold episodes, each subject rated the six predefined symptoms during each episode for five days. The symptom "general malaise" was excluded from summation, because it has been shown that this parameter was unspecific and was without group difference in $95 \%$ of all the episodes $\left(\mathrm{p}_{\gamma^{2}}=0.818\right)$.

Table 5 summarizes the total score during the course of the episodes. It is obvious that the total scores were significantly lower in the AG than in the PG at all stages of the episodes.

For the three typical cold symptoms "sore throat

Table 3. Number of common cold episodes during common cold episode season (13 weeks) in the intention to treat (ITT) and per protocol (PP) population.

\begin{tabular}{ccccc}
\hline \multirow{2}{*}{$\begin{array}{c}\text { Number of } \\
\text { episodes }\end{array}$} & $\mathrm{AG}(\mathrm{ITT} \mathrm{n}=45 / \mathrm{PP} \mathrm{n}=41)$ & \multicolumn{2}{c}{$\mathrm{PG}(\mathrm{ITT} \mathrm{n}=49 / \mathrm{PP} \mathrm{n}=44)$} \\
\cline { 2 - 5 } & Number & $\%$ & Number & $\%$ \\
\hline 0 & $13 / 13$ & $28.9 / 31.7$ & $6 / 6$ & $12.2 / 13.6$ \\
1 & $20 / 18$ & $44.4 / 43.9$ & $20 / 20$ & $40.8 / 45.5$ \\
2 & $9 / 7$ & $20.0 / 17.1$ & $18 / 15$ & $36.7 / 34.1$ \\
3 & $3 / 3$ & $6.7 / 7.3$ & $4 / 2$ & $8.2 / 4.5$ \\
4 & $0 / 0$ & $0 / 0$ & $1 / 1$ & $2.0 / 2.3$ \\
total & $47 / 41$ & $100 / 100$ & $72 / 60$ & $99.9 / 100$ \\
$\mathrm{p}_{\mathrm{u}}$ & \multicolumn{4}{c}{$0.020 / 0.045$} \\
\hline
\end{tabular}

$\mathrm{p}_{\mathrm{u}}$ : p-value for total number of episodes; AG: active group; PG: placebo group.

Table 4. Mean common cold episodes after 13 and 26 weeks (intention to treat population).

\begin{tabular}{ccccc}
\hline \multirow{4}{*}{ Time point } & \multicolumn{5}{c}{ Common cold episodes } \\
\cline { 2 - 5 } & $\begin{array}{c}\text { Number of } \\
\text { Episodes }\end{array}$ & $A G$ & $P G$ & $p_{\text {u}}$-value \\
\hline \multirow{2}{*}{ 13 weeks } & Total & 47 & 72 & 0.020 \\
& Average \pm SD & $1.04 \pm 0.88$ & $1.47 \pm 0.89$ & 0.022 \\
26 weeks & Total & 76 & 95 & 0.406 \\
& Average \pm SD & $1.69 \pm 1.08$ & $1.94 \pm 1.13$ & 0.277 \\
\hline
\end{tabular}

pu: p-value obtained by U-test; AG: active group; PG: placebo group; SD: standard deviation. and/or difficulty swallowing", "hoarseness and/or cough", and "runny nose", a significantly higher success (defined as being complaint-free during the first five days of an episode) was shown in the $\mathrm{AG}(\mathrm{p}=0.034$, respectively $<$ 0.0001 , respectively 0.001 ). These three typical cold symptoms and the total cold sum score were significantly less pronounced at the beginning of the episodes in the AG than in the PG $\left(\mathrm{p}_{x^{2}}=0.011\right.$ to 0.046 , respectively $\mathrm{p}_{\mathrm{u}}=0.029$ ).

The results of the secondary objectives clearly demonstrate that the prophylactic intake of beta-glucan led to milder common cold symptoms in case of an infection episode.

\subsection{Global Evaluation of the Efficacy during Episodes (ITT)}

Global evaluation of the efficacy after each episode was judged as "very good" or "good" for $74.1 \%$ of participants of the active treatment and for $48.3 \%$ of participants of the placebo treatment, as assessed by physicians, and for $76.6 \%(49.4 \%)$ of participants, as assessed by participants. Both participants and physicians rated the efficacy of the beta-glucan preparation significantly better than the placebo $\left(\mathrm{p}_{\chi^{2}}=0.001, \mathrm{p}_{\chi^{2}}=0.002\right.$, respectively; Table 6).

\subsection{Safety Evaluation}

All measured clinical parameters, body weight, temperature, heart rate and blood pressure remained almost constant during the study, with no significant differences between the two study populations.

The global assessment of tolerability for both treatments was rated at the end of the study as "very good" by approximately $2 / 3$ of participants and physicians, or as "good" by approximately $1 / 3$ of participants and physicians with no group differences $\left(\mathrm{p}_{\chi^{2}}=0.568, \mathrm{p}_{\chi^{2}}=\right.$ 0.395 , respectively).

\section{Adverse Events}

A total of six adverse events occurred during this nutritional study. Four of them occurred in the AG (gastrointestinal infection, hypertonic blood pressure, pain in lumbar spine region during physical activity, pain/ swelling of the upper ankle joint after playing soccer) and two in the PG (cut into finger, pain in the knee joint). All of them were "not severe", did not lead to termination of the study, and were judged as being "unlikely" connected to the intake of the investigational product. The two study groups did not differ in the proportion of subjects with adverse events (AG: $8.0 \%$ vs PG: $4.0 \%$; $\left.\mathrm{p}_{\gamma^{2}}=0.4\right)$. None of the adverse events led to a preterm drop-out. 
Table 5. Total score values in the course of the common cold episodes.

\begin{tabular}{cccccc}
\hline & \multicolumn{5}{c}{ Common cold score } \\
\cline { 2 - 6 } & Day 1 mean $\pm S D$ & Day 2 mean $\pm S D$ & Day 3 mean $\pm S D$ & Day 4 mean $\pm S D$ & Day 5 mean $\pm S D$ \\
\hline Total & $5.1 \pm 2.3$ & $5.3 \pm 2.4$ & $4.2 \pm 2.4$ & $2.9 \pm 2.4$ & $1.7 \pm 2.0$ \\
AG & $4.6 \pm 2.1$ & $4.8 \pm 2.3$ & $3.5 \pm 2.1$ & $2.4 \pm 1.9$ & $1.4 \pm 1.6$ \\
PG & $5.5 \pm 2.6$ & $5.7 \pm 2.4$ & $4.8 \pm 2.5$ & $3.4 \pm 2.6$ & $2.0 \pm 2.3$ \\
p $_{\text {-value }}$ & 0.029 & 0.014 & 0.001 & 0.015 & 0.059 \\
p $_{\mathrm{t}}$-value & 0.018 & 0.009 & 0.001 & 0.005 & 0.034 \\
\hline
\end{tabular}

$\mathrm{p}_{\mathrm{u}}$ or $\mathrm{p}_{\mathrm{t}}$ : $\mathrm{p}$-value obtained by U-test or t-test; AG: active group; PG: placebo group; SD: standard deviation.

Table 6. Global evaluation of the efficacy by the investigators and participants.

\begin{tabular}{cccccc}
\hline \multirow{2}{*}{ Group } & \multicolumn{5}{c}{ Evaluation [\%] } \\
\cline { 2 - 6 } & Verygood & Good & Moderate & Poor & p-value \\
\hline By investigators & & & 3.9 & 0.002 \\
AG & 24.7 & 49.4 & 22.1 & 20.2 & \\
PG & 20.2 & 28.1 & 31.5 & & \\
By patients & & & & & \\
AG & 23.4 & 53.2 & 19.5 & 3.9 & 0.001 \\
PG & 20.2 & 29.2 & 31.5 & 19.1 & \\
\hline
\end{tabular}

$\mathrm{p}_{x^{2}}$ : p-value for distribution; AG: active group; PG: placebo group.

\section{Discussion}

During this placebo-controlled, randomized, nutritional study, the effect of an insoluble yeast beta-glucan on the body's resistance against invading pathogens was investigated. The common cold was used as a model system. Over a period of 26 weeks, the number of common cold infections was evaluated in subjects with recurring common colds. The beta-glucan group had significantly fewer subjects without infections (AG $15.6 \%$ vs PG $2.0 \% ; p=0.019)$, and $20 \%$ fewer infections compared to placebo ( $\mathrm{AG} \mathrm{n}=76$ versus $\mathrm{PG} \mathrm{n}=95$ infection), but both groups did not differ statistically regarding the total number of infections $(\mathrm{p}=0.406)$.

However, if the data of the first half of the study (first 13 weeks - which was during the most intense infection time) are analyzed, then the beta-glucan group has significantly fewer infections than the PG group. During this time of intense infection, $69.5 \%$ of the infections occurred (119 of 171) from which only 47 occurred in the beta-glucan group and 72 in the PG group $(p=0.02)$. The low incidence of common colds during the second half of the study, March till June, cannot be explained by the efficacy of beta glucans on the subjects in the active group, since the number of infections occurring in the placebo group was equal to the number of infections in the active group. This reduction of common cold episodes might be caused by higher temperatures and generally increased well-being of humans during spring and summer time. Thus, the greatest effect of Yestimun ${ }^{\circledR}$ on the primary outcome variable, which was the number of common cold infections, was observed during the winter season.

One limitation of the study was the number of participants included into this nutritional being determined to be 50 per group, due to missing previous product-specific studies. Furthermore, the included subjects had to be susceptible to common cold (at least three within the last six months). However, the subjects of the PG had fewer episodes than expected (mean number of infections in PG: 1.94 within 26 weeks). The small number of participants, together with the lower rate of actual infection, and the realization of the second study half during spring and summer time were probably responsible for the failure to show statistical significance regarding the total number of infections during the whole study duration. Therefore, the effects observed in this study have to be confirmed in a larger scale study exclusively during the winter period with increased risk of common cold.

Several investigations using other beta-glucan preparations to prevent the risk of common cold infections have already been published.

In one study, the effect of beta-glucan preparations has been investigated on the respiratory tract of athletes [15]. Enhanced exercises seem to weaken the immune system and may cause increased risk of upper respiratory tract infections $[16,17]$. In the study by Talbott, marathon runners were treated with either 250 or $500 \mathrm{mg}$ of a $\beta-1,3$ / 1,6-glucan preparation from Saccharomyces cerevisiae (Wellmune $\mathrm{WPG}^{\circledR}$ ) for four weeks post-marathon. Both beta-glucan groups reported significantly fewer infections symptoms and better overall health, compared to placebo. While only $8 \%$ of the subjects of both betaglucan groups reported infections at week four, $24 \%$ of 
the placebo group subjects reported infection symptoms. Furthermore, the subjects reported better overall health and a more positive mood state compared to placebo four weeks after completing the marathon [15]. In another study, the effect of a beta-glucan preparation made from Pleurotus ostreatus together with vitamin $\mathrm{C}$ on the cellular immune response and upper respiratory tract infections (URTI) was investigated on athletes of different sports disciplines [18]. Fifty athletes took either two capsules containing $100 \mathrm{mg}$ beta-glucan and $100 \mathrm{mg}$ vitamin $\mathrm{C}$ or $100 \mathrm{mg}$ vitamin $\mathrm{C}$ alone for three months. In that trial, the beta-glucan-treated athletes reported significantly less URTI than the vitamin C group (65 versus 117 episodes) [18]. In one clinical trial lasting 12 weeks [19], the effect of beta-glucan $(500 \mathrm{mg} /$ day $)$ from $\mathrm{Sac}$ charomyces cerevisiae (Wellmune $\mathrm{WPG}^{\mathbb{R}}$ ) was compared to placebo in healthy subjects. In that study, the investigators found no difference in the incidence of common cold infections. Even though the number of infections was not different, the symptoms strength was reduced in the beta-glucan group since none of the subjects missed school or work due to colds, while the placebo group did so on average for 1.38 days.

The results of these above cited studies cannot be directly compared to our study, since time courses, applied dosages, subject populations or measured outcomes are not comparable. However, subjects treated with the above-described beta-glucan preparation had fewer infections or reduced symptoms, like in the study presented here. All these studies were made with insoluble beta-glucan preparations.

It is known that various beta-glucan preparations have different effects on the immune system, where not only the size and the degree of branching are responsible but also the solubility $[7,9,20]$. One explanation could be the fact that for activation of the Dectin-1 receptor, whole beta-glucans are necessary, as shown very recently. Smaller size particles or soluble beta-glucans are inactive, despite efficient binding to the receptor [21]. Based on this result, Goodridge et al. [21] hypothesized "that a larger molecule may be required to provide a greater degree of receptor crosslinking to permit activation".

Beta-glucan is thought to strengthen the immune system by activation of innate and adaptive immune response mechanisms [2]. Various other nutritional and herbal agents have been investigated for their immune system strengthening abilities. One of them is Echinacea, commonly used for prevention of common cold infection and known for its immune system stimulating abilities. Its efficacy, however, is discussed controversially [2224]. Due to the fact that all of the intervention trials differ in study collectives, time courses and primary and secondary outcomes it is not possible to directly compare the effect of Echinaceae preparations to the preventive effect of beta-glucan. In one trial, the effect of an Echinaceae preparation was also investigated on subjects with recurring infections [25]. However, the treatment duration was only 8 weeks. Within that period, $25.9 \%$ of the placebo-treated subjects were free from infections compared to $35.2 \%$ of the active group. Based on this data, the effect size was calculated to be $9.3 \%$ (difference Echinacea treatment versus placebo). Similar results were also observed by Grimm and Müller [26]. During their two months study, they observed $35 \%$ of Echinaceae treated subjects without infections and $26 \%$ of the placebo treated subjects in a group of subjects with recurring infection (effect size 9\%). This data can be compared to our data of the first 15 weeks of the study, where $28.9 \%$ of the AG and $12.2 \%$ of the PG were free from infection, which represents an effect size of $16.7 \%$. Based on these results, we conclude that beta-glucan might be at least as effective as Echinaceae in the prevention of common colds. This, however, has to be shown in a comparative two-armed study where both preparations are compared directly.

As another treatment to prevent common cold, high dosages of vitamin $\mathrm{C}$ are frequently used. However, in the Cochrane review [27], the author came to the conclusion that high intakes of Vitamin $\mathrm{C}$ do not reduce the incidence of episodes in the normal population. Only people with high physical stress (athletes or soldiers) might benefit.

Zinc is also important for the normal function of the immune system. When taken within 24 hours of onset of a cold episode, the severity of symptoms and the duration of the common cold is reduced. Further, when zinc is supplemented for at least 5 months, the rate of incidences as well the prescription of antibiotics in children is reduced [28]. However, it will not prevent common cold episodes in the normal, non-deficient population.

Both substances, vitamin $\mathrm{C}$ and zinc, are undeniably important for the function of our immune system. However, it seems that only subpopulations with a deficiency of these substances may profit from supplementation, which then reduces the incidences of cold episodes.

The insoluble beta-glucan preparation made of brewers' yeast used in this study was well tolerated when taken for 26 weeks. Neither placebo nor beta-glucan showed any difference in the number of observed adverse events $(p=0.4)$, from which none was judged to be treatment related. All the measured clinical parameters (body weight, temperature, heart rate and blood pressure) remained almost constant in both groups during the study. Approximately $2 / 3$ of the subjects evaluated the safety in agreement with the investigators, without a group difference, as "very good" and about $1 / 3$ as "good". Consider- 
ing the six terminations and nine study plan violations, compliance (intake of the capsules, adherence to the study period) of the subjects was assessed as good for this six months nutritional study. The overall good compliance demonstrates the consumers' acceptance of the study product.

In conclusion, the present study demonstrates a prophylactic effect of an insoluble yeast beta-glucan preparation on the occurrence of common colds as opposed to placebo during seasons with increased common cold incidences. When episodes occurred, they were from the beginning less pronounced and subsided faster. This result, however, has to be confirmed by further investigations with an appropriate sample size.

\section{Acknowledgements}

The study was funded by Leiber GmbH (Bramsche, Germany). The authors declare no conflict of interest.

\section{REFERENCES}

[1] B. Blattberg, "Increase in Bactericidal Activity as a Result of Injection of Zymosan," Proceedings of the Society for Experimental Biology and Medicine, Vol. 92, No. 4, 1956, pp. 745-748.

[2] G. C. Chan, W. K. Chan and D. M. Sze, "The Effects of Beta-Glucan on Human Immune and Cancer Cells," Journal of Hematology \& Oncology, Vol. 2, No. 2009, 2009, p. 25.

[3] D. B. Zekovic, S. Kwiatkowski, M. M. Vrvic, D. Jakovljevic and C. A. Moran, "Natural and Modified (13)-Beta-D-Glucans in Health Promotion and Disease Alleviation," Critical Reviews in Biotechnology, Vol. 25, No. 4, 2005, pp. 205-230. doi:10.1080/07388550500376166

[4] L. G. Fleischer, G. Gerber, G. Gremmels, H.-D. Lippert and G. Westphal, "Experimentelle Modelluntersuchungen zum Einsatz von $(1,3),(1,6)$-Beta-D-Glucan aus Saccharomyces Cerevisiae Fibona Health Products GmbH," 2001.

[5] J. Li, J. Xing, D. Li, X. Wang, L. Zhao, S. Lv and D. Huang, "Effects of Beta-Glucan Extracted from Saccharomyces Cerevisiae on Humoral and Cellular Immunity in Weaned Piglets," Archives of Animal Nutrition, Vol. 59, No. 5, 2005, pp. 303-312. doi:10.1080/17450390500247832

[6] D. Akramiene, A. Kondrotas, J. Didziapetriene and E. Kevelaitis, "Effects of Beta-Glucans on the Immune System," Medicina, Vol. 43, No. 8, 2007, pp. 597-606.

[7] J. A. Bohn and J. N. BeMiller, "(1-3)- $\beta$-D-Glucans as Biological Response Modifiers: A Review of StructureFunctional Activity Relationships," Carbohydrate Polymers, Vol. 28, 1995, pp. 3-14. doi:10.1016/0144-8617(95)00076-3

[8] T. Kojima, K. Tabata, W. Itoh and T. Yanaki, "Molecular
Weight Dependence of the Antitumor Activity of Schizophyllan," Agricultural Biology and Chemistry, Vol. 50, No. 1, 1986, pp. 231-232. doi:10.1271/bbb1961.50.231

[9] E. Sonck, B. Devriendt, B. Goddeeris and E. Cox, "Varying Effects of Different Beta-Glucans on the Maturation of Porcine Monocyte-Derived Dendritic Cells," Clinical and Vaccine Immunology, Vol. 18, No. 9, 2011, pp. 14411446. doi:10.1128/CVI.00080-11

[10] D. C. Nieman, D. A. Henson, M. McMahon, J. L. Wrieden, J. M. Davis, E. A. Murphy, S. J. Gross, L. S. McAnulty and C. L. Dumke, "Beta-Glucan, Immune Function, and Upper Respiratory Tract Infections in Athletes," Medicine and Science in Sports and Exercise, Vol. 40, No. 8, 2008, pp. 1463-1471. doi:10.1249/MSS.0b013e31817057c2

[11] J. Malaczewska, R. Wójcik, L. Jung and A. K. Siwicki, "Effect of Biolex $\beta$-HP on Selected Parameters of Specific and Non-Specific Humoral and Cellular Immunity in Rats," Bulletin of the Veterinary Institute in Pulawy, Vol. 54, No. 1, 2010, pp. 75-80.

[12] R. Wojcik, "Effect of Biolex Beta-HP on Phagocytic Activity and Oxidative Metabolism of Peripheral Blood Granulocytes and Monocytes in Rats Intoxicated by Cyclophosphamide," Polish Journal of Veterinary Sciences, Vol. 13, No. 1, 2010, pp. 181-188.

[13] R. Wójcik, E. Janowska, J. Malaczewska and A. K. Siwicki, "Effect of $\beta-1,3 / 1,6$-D-glucan on the Phagocytic Activity and Oxidative Metabolism of Peripheral Blood Granulocytes and Monocytes in Rats," Bulletin of the Veterinary Institute in Pulawy, Vol. 53, No. 2, 2009, pp. 241-246.

[14] R. Eccles, "Understanding the Symptoms of the Common Cold and Influenza," Lancet Infectious Diseases, Vol. 5, No. 11, 2005, pp. 718-725. doi:10.1016/S1473-3099(05)70270-X

[15] S. Talbott and J. Talbott, "Effect of BETA 1,3/1,6 GLUCAN on Upper Respiratory Tract Infection Symptoms and Mood State in Marathon Athlets," Journal of Sports Science and Medicine, Vol. 8, 2009, pp. 509-515.

[16] D. C. Nieman, "Risk of Upper Respiratory Tract Infection in Athletes: An Epidemiologic and Immunologic Perspective," Journal of Athletic Training, Vol. 32, No. 4, 1997, pp. 344-349.

[17] N. P. Walsh, M. Gleeson, R. J. Shephard, J. A. Woods, N. C. Bishop, M. Fleshner, C. Green, B. K. Pedersen, L. Hoffman-Goetz, C. J. Rogers, H. Northoff, A. Abbasi and P. Simon, "Position Statement. Part One: Immune Function and Exercise," Exercise Immunology Review, Vol. 17, 2011, pp. 6-63.

[18] K. Bergendiova, E. Tibenska and J. Majtan, "Pleuran (Beta-Glucan from Pleurotus ostreatus) Supplementation, Cellular Immune Response and Respiratory Tract Infections in Athletes," European Journal of Applied Physiology, Vol. 111, No. 9, 2011, pp. 2033-2040. doi:10.1007/s00421-011-1837-z

[19] S. Feldman, H. I. Schwartz, D. S. Kalman, A. Mayers, H. M. Kohrman, R. Clemens and D. Krieger, "Randomized Phase II Clinical Trials of Wellmune WGP for Immune 
Support during Cold and Flu Season," Journal of Applied Research, Vol. 9, No. 1-2, 2009, pp. 30-42.

[20] P. J. Rice, E. L. Adams, T. Ozment-Skelton, A. J. Gonzalez, M. P. Goldman, B. E. Lockhart, L. A. Barker, K. F. Breuel, W. K. Deponti, J. H. Kalbfleisch, H. E. Ensley, G. D. Brown, S. Gordon and D. L. Williams, "Oral Delivery and Gastrointestinal Absorption of Soluble Glucans Stimulate Increased Resistance to Infectious Challenge," Journal of Pharmacology and Experimental Therapeutics, Vol. 314, No. 3, 2005, pp. 1079-1086. doi:10.1124/jpet.105.085415

[21] H. S. Goodridge, C. N. Reyes, C. A. Becker, T. R. Katsumoto, J. Ma, A. J. Wolf, N. Bose, A. S. Chan, A. S. Magee, M. E. Danielson, A. Weiss, J. P. Vasilakos and D. M. Underhill, "Activation of the Innate Immune Receptor Dectin-1 upon Formation of a 'Phagocytic Synapse'," Nature, Vol. 472, No. 7344, 2011, pp. 471-475. doi:10.1038/nature10071

[22] R. Schoop, P. Klein, A. Suter and S. L. Johnston, "Echinacea in the Prevention of Induced Rhinovirus Colds: A Meta-Analysis," Clinical Therapeutics, Vol. 28, No. 2, 2006, pp. 174-183. doi:10.1016/j.clinthera.2006.02.001

[23] S. A. Shah, S. Sander, C. M. White, M. Rinaldi and C. I. Coleman, "Evaluation of Echinacea for the Prevention and Treatment of the Common Cold: A Meta-Analysis,"
Lancet Infectious Diseases, Vol. 7, No. 7, 2007, pp. 473480. doi:10.1016/S1473-3099(07)70160-3

[24] D. S. Senchina, N. B. Shah, D. M. Doty, C. R. Sanderson and J. E. Hallam, "Herbal Supplements and Athlete Immune Function-What's Proven, Disproven, and Unproven?" Exercise Immunology Review, Vol. 15, 2009, pp. 66-106.

[25] D. Schöneberger, "Immunstimulierende Wirkung von Echinacin Madaus Liquidum auf Verlauf und Schweregrad von Erkältungskrankheiten," Forum Immunologie, Vol. 8, 1996, pp. 2-11.

[26] W. Grimm and H. Müller, "A Randomized Controlled Trial of the Effect of Fluid Extract of Echinacea Purpureaz on the Indicence and Severity of Colds and Respiratory Infections," American Journal of Medicine, Vol. 109, 1999, pp. 138-143. doi:10.1016/S0002-9343(98)00406-9

[27] R. M. Douglas, H. Hemila, E. Chalker and B. Treacy, "Vitamin C for Preventing and Treating the Common Cold," Cochrane Database of Systematic Reviews, Vol. 3, 2007, Article ID: CD000980.

[28] M. Singh and R. R. Das, "Zinc for the Common Cold," Cochrane Database of Systematic Reviews, Vol. 2, 2011, Article ID: CD001364. doi:10.1002/14651858.CD001364.pub3 